\title{
Fetal signalling initiates parturition
}

Parturition is thought to be initiated by an increased inflammatory response and expression of contractile proteins in the uterine tissues, but the underlying mechanism is unclear. Pulmonary surfactant-associated protein-A (SP-A), which is secreted from the fetal lungs is thought to inititate this process. Now, new data indicate that nuclear receptor coactivators 1 and 2 (also known as steroid receptor coactivators; SRC-1 and SRC-2), regulates transcription of SP-A and consequently induction of labour.

In mice with heterozygous mutations in both SRC-1 and SRC-2 (SRC-1/-2 dhet), which avoids the lethality seen in double knockout mice, parturition was delayed by $\sim 38 \mathrm{~h}$ compared with wild-type mice. Interestingly, this delay was also seen in wild-type mice with $S R C$-1/-2 deficient embryos, which suggests that the signal to initiate labour is derived from the fetus.

Both SRC-1/-2 dhet and wild-type mothers carrying $S R C-1 /-2$ deficient embryos had fewer markers of inflammation in the myometrium, reduced expression of genes associated with uterine contraction and higher circulating levels of progesterone than wild-type mothers carrying wild-type embryos. Similarly, levels of SP-A and lysophosphatidylcholine acyltransfersase-1, an enzyme that produces proinflammatory glycerophospholipid platelet-activating factor (PAF), which might induce labour, were reduced in the lungs of SRC-1/-2 deficient fetuses. Importantly, the delay in parturition was rescued by injection of SP-A or PAF into amniotic fluid of SRC-1/-2 dhet mice carrying SRC-1/-2 deficient embryos. Taken together, these results suggest that signals from the lungs of fetuses can initiate labour.

Tim Geach

Original article Gao, L. et al. Steroid receptor coactivators 1 and 2 mediate fetal-to-maternal signaling that initiates parturition. J. Clin. Invest. doi:10.1172/ JCI78544 